\title{
Development and Validation of UV-Visible Spectrophotometric Baseline Manipulation Method for Simultaneous Quantitation of Tenofovir Disoproxil Fumarate and Emtricitabine in Pharmaceutical Dosage Form
}

\author{
Vishnu P. Choudhari, Sanket R. Parekar, Subhash G. Chate, Pradeep D. Bharande, \\ Rajiv R. Singh, and Bhanudas S. Kuchekar
}

Pharmacy Department, Maharashtra Institute of Pharmacy, MIT Campus, Paud Road, Kothrud, Pune 411038, India

Correspondence should be addressed to Vishnu P. Choudhari; viraj1404@rediffmail.com

Received 21 June 2012; Accepted 20 July 2012

Academic Editor: Lahcen Bih

Copyright (C) 2013 Vishnu P. Choudhari et al. This is an open access article distributed under the Creative Commons Attribution License, which permits unrestricted use, distribution, and reproduction in any medium, provided the original work is properly cited.

\begin{abstract}
A simple, economical, precise, and accurate new UV-visible spectrophotometric baseline manipulation method for simultaneous determination of tenofovir disoproxil fumarate (TE) and emtricitabine (EM) in combined tablet dosage form has been developed. The method is based on baseline manipulation (difference) spectroscopy where amplitudes at 261 and 289.9 nm were selected to determine TE and EM, respectively, in combined formulation, and distilled water was used as solvent. Both drugs obey Beer's law in the concentration ranges of $4-20 \mu \mathrm{g} / \mathrm{mL}$ for TE and $6-30 \mu \mathrm{g} / \mathrm{mL}$ for EM. The results of analysis have been validated statistically, and recovery studies confirmed the accuracy of the proposed method which was carried out by following the ICH guidelines.
\end{abstract}

\section{Introduction}

Tenofovir (TE): 9[(R)2[[bis[[(isopropoxycarbonyl)oxy]methoxy]phosphinyl]methoxy]propyl] and Emtricitabine (EM): 5-fluro-1-(2R, 5S)-[2-9-hydroxymethyl]-1,3-oxathiolan-5-yl both are the antiviral agents. Pharmacological and literature survey reveals that there are various methods reported for estimation of titled analytes individually and in combination. Ratio derivative methods, first order derivative, absorbance corrected [1], area under curve, dual wavelength [2], simultaneous equation, and Iso-absorptive point UVspectrophotometric methods [3] were developed for the same combinations in the institute, therefore the same combination was selected to develop and validate the baseline manipulation method, which is simple and require less number of standard solutions and economical as compared to reported methods. Therefore, the aim of the study was to develop and validate new analytical baseline manipulation methodology and statistically compare the results of the proposed method with reported methods.
Validated computer system, MIP Pharmasoft 1.0, was used for calculation purpose. The proposed method was validated as per the International Conference on Harmonization (ICH) guidelines [4].

\section{Materials and Methods}

2.1. Materials and Reagents. Pure drug sample of TE, purity $99.86 \%$ and EM, purity $99.92 \%$ was kindly supplied as a gift sample by Emcure Pharmaceutical Pvt., Ltd., Pune. These samples were used without further purification. Tablet used for analysis was TENVIR-EM (Batch no. X81241) manufactured by Cipla Ltd., Goa, India, containing TE $300 \mathrm{mg}$ and EM 200 mg per tablet.

2.2. Instrumentation. An UV-visible double beam spectrophotometer (Varian Cary 100) with $10 \mathrm{~mm}$ matched quartz cells was used. All weighing were done on electronic balance (Model Shimadzu AUW-220D). 


\section{Methods}

3.1. Preparation of Standard Stock Solutions and Calibration Curve. Standard stock solution containing $200 \mu \mathrm{g} / \mathrm{mL}$ of TE and $300 \mu \mathrm{g} / \mathrm{mL}$ of EM was prepared separately in the distilled water. Individual working standard solution containing $20 \mu \mathrm{g} / \mathrm{mL}$ of TE was prepared from standard stock solution. Mixed standard solutions containing TE $(12 \mu \mathrm{g} / \mathrm{mL})$ and EM $(18 \mu \mathrm{g} / \mathrm{mL})$ were prepared by using standard stock solutions. The mixed standard solutions of these drugs containing $4-20 \mu \mathrm{g} / \mathrm{mL}$ of TE and $6-30 \mu \mathrm{g} / \mathrm{mL}$ of EM were prepared by serial dilutions of standard stock solutions in distilled water. Mixed standard solutions were scanned using $20 \mu \mathrm{g} / \mathrm{mL}$ of TE solution as blank. Instrument response at $261 \mathrm{~nm}$ and $289.9 \mathrm{~nm}$ was measured for TE and EM, respectively, and was used to prepare calibration curve. Six replicates of five mixed standard solutions were used to prepare calibration curve. As the correlation coefficient is not true indicator of linearity, Fischer variance ratio (test of linearity) was used to study the linearity of the method [5]. Test of linearity was performed by using MIP Pharmasoft 1.0, software developed and validated at MAEER'S Maharashtra Institute of Pharmacy, Pune. Absorbance of spectrum, by using the above mentioned procedure, was used to prepare calibration curves for both drugs by baseline manipulation (difference) method. Absorbance values at selected wavelengths of the curve were used to construct equations of line ((1) for TE and EM, resp.) which were used to calculate amount of analytes in unknown samples. Beer's law obeyed in the concentration range of 4-20 $\mu \mathrm{g} / \mathrm{mL}$ for TE and 6-30 $\mu \mathrm{g} / \mathrm{mL}$ for EM as follows:

$$
\begin{gathered}
C_{\mathrm{TE}}=\frac{\left(A_{\mathrm{TE}}-I_{\mathrm{TE}}\right)}{S_{\mathrm{TE}}}, \\
C_{\mathrm{EM}}=\frac{\left(A_{\mathrm{EM}}-I_{\mathrm{EM}}\right)}{S_{\mathrm{EM}}},
\end{gathered}
$$

where, $I_{\mathrm{TE}}$ and $I_{\mathrm{EM}}$ are the intercepts of lines of TE and EM, respectively. $S_{\mathrm{TE}}$ and $S_{\mathrm{EM}}$ are slopes of lines of $\mathrm{TE}$ and EM, respectively. $A_{\mathrm{TE}}$ and $A_{\mathrm{EM}}$ are the absorbance values of formulation at $261 \mathrm{~nm}$ and $289.9 \mathrm{~nm}$ for TE and $\mathrm{EM}$, respectively. $C_{\mathrm{TE}}$ and $C_{\mathrm{EM}}$ are the concentrations in $\mu \mathrm{g} / 100 \mathrm{~mL}$ of TE and EM, respectively.

3.2. Analysis of Tablet Formulation. Twenty tablets were weighed accurately and a quantity of tablet powder equivalent to $200 \mathrm{mg}$ of TE (300 mg of EM) was weighed and dissolved in the $80 \mathrm{~mL}$ of distilled water with the aid of ultrasonicator for $15 \mathrm{~min}$, and solution was filtered through Whatman paper no. 41 into a $100 \mathrm{~mL}$ volumetric flask. Filter paper was washed with the distilled water, adding washings to the volumetric flask and volume was made up to mark. The solution was suitably diluted with distilled water to get $200 \mu \mathrm{g} / \mathrm{mL}$ of $\mathrm{TE}$ and $300 \mu \mathrm{g} / \mathrm{mL}$ of EM. Instrument response for the analytes was measured by following the procedure described in preparation of standard stock solutions and calibration curve.

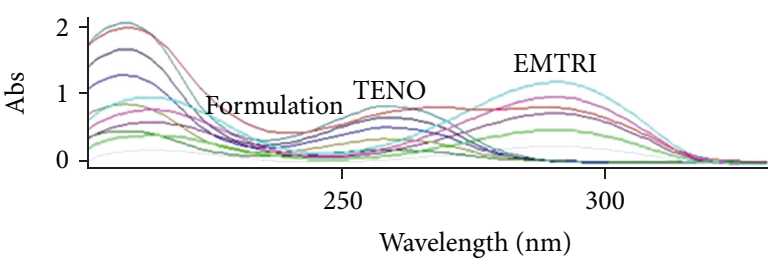

FIGURE 1

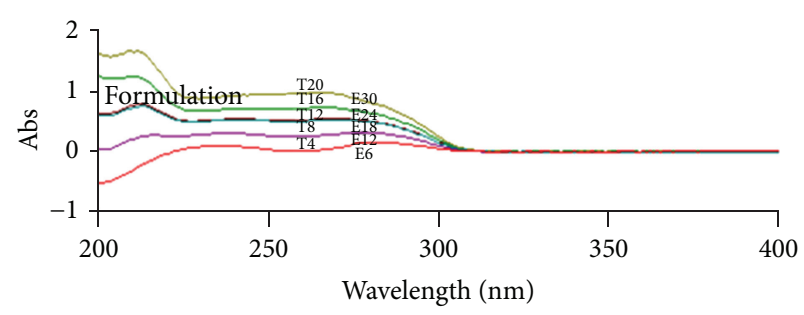

Figure 2

3.3. Accuracy. The accuracy of the assay method was evaluated with the recovery of the standards from excipients. Recovery studies were carried out by applying the method to drug content present in tablet dosage form to which known amount of mix standard of TE and EM were added at $50 \%, 100 \%$, and $150 \%$ levels. At each of the levels, three determinations were performed and results were obtained.

3.4. Precision of the Method. The precision of repeatability was studied by six replicate analyses of tablet solutions containing 12 and $18 \mu \mathrm{g} / \mathrm{mL}$ of TE and EM, respectively. The precision was also studied in terms of intraday changes in absorbance of drug solution on the same day and on three different days. The intraday precision of the developed method was determined by preparing the tablet samples of the same batch in nine determinations with three concentrations and three replicates each on same day. The interday precision was also determined by assaying the tablets in triplicate per day for consecutive 3 days. The intraday and interday variation was calculated in terms of percentage relative standard deviation. Precision of analyst was determined by repeating the method by another analyst working in the lab. Three concentration used for the study were 12, 16, and $20 \mu \mathrm{g} / \mathrm{mL}$ of TE and 18,24 , and $30 \mu \mathrm{g} / \mathrm{mL}$ of EM. Precision data was also statistically evaluated by using experimental design based on ANOVA.

3.5. Method Sensitivity (LOD and LOQ). The values of LOD and LOQ were calculated by using $\sigma$ (standard deviation of response) and $b$ (slope of the calibration curve) and by using equations as $\operatorname{LOD}=(3.3 \times \sigma) / b$ and $\operatorname{LOQ}=(10 \times \sigma) / b$.

3.6. Robustness. To determine the robustness of the method, the final experimental conditions were purposely altered and the results were examined. The parameters considered $( \pm$ values) for the study were, shaking time of solution ( $\pm 2 \mathrm{~min})$, 
TABLE 1: Optical characteristics of the method and result of formulation analysis, recovery study, and method sensitivity.

\begin{tabular}{lcc}
\hline Parameter & & Analyte \\
& TE & 289.9 \\
$\lambda$ max $(\mathrm{nm})$ & 261 & $6-30$ \\
Range $(\mu \mathrm{g} / \mathrm{mL})$ & $4-20$ & \\
Regression equation $Y=m x+c$ & 0.05974 & 0.022127 \\
$\quad$ Slope $(\mathrm{m})$ & -0.23012 & -0.01772 \\
$\quad$ Intercept $(c)$ & 0.999 & 0.999 \\
Correlation coefficient & 1.58 & 4.5 \\
Limit of quantitation (LOQ) & 0.55 & 1.5 \\
Limit of detection (LOD) & & \\
Formulation analysis (\% assay, \% RSD) & $99.75,0.84$ & $99.35,1.01$ \\
$\quad$ F I & $101.2,1.23$ & $100.2,0.97$ \\
F II & & \\
Recovery study at the level (\% R, \% RSD) & $100.21,0.54$ & $101.4,0.75$ \\
$50 \%$ & $100.4,0.94$ & $99.78,0.69$ \\
$100 \%$ & $99.45,0.54$ &
\end{tabular}

TABLE 2: Result of recovery studies for TE and EM.

\begin{tabular}{|c|c|c|c|c|}
\hline \multirow[b]{2}{*}{ Analyte name } & \multicolumn{2}{|c|}{ Formulation study $(n=6)$} & \multicolumn{2}{|c|}{ Recovery (accuracy) study } \\
\hline & $\begin{array}{c}\text { Base level amount } \\
\mu \mathrm{g} / \mathrm{mL}\end{array}$ & $\begin{array}{c}\text { Amount spiked } \\
\mu \mathrm{g} / \mathrm{mL}\end{array}$ & Recovery level & $\begin{array}{c}\% \text { Recovery, } \\
\% \operatorname{RSD}(n=3)\end{array}$ \\
\hline \multirow{3}{*}{$\mathrm{TE}$} & 6 & 3 & $50 \%$ & $100.21,0.54$ \\
\hline & 6 & 6 & $100 \%$ & $100.4,0.94$ \\
\hline & 6 & 9 & $150 \%$ & $99.45,0.54$ \\
\hline \multirow{3}{*}{ EM } & 9 & 4.5 & $50 \%$ & $99.5,0.45$ \\
\hline & 9 & 9 & $100 \%$ & $101.4,0.75$ \\
\hline & 9 & 13.5 & $150 \%$ & $99.78,0.69$ \\
\hline
\end{tabular}

TABLE 3: Intraday precision study for TE.

\begin{tabular}{|c|c|c|c|}
\hline \multirow{2}{*}{ Analysts } & \multicolumn{3}{|c|}{ Time } \\
\hline & $T 1$ & $T 2$ & T3 \\
\hline Analyst 1 & 99.5 & 100.3 & 99.34 \\
\hline Analyst 1 & 100.2 & 100.4 & 99.54 \\
\hline Analyst 1 & 101.1 & 101.1 & 100.4 \\
\hline Analyst 2 & 99.21 & 99.54 & 101.3 \\
\hline Source of variation & Sum of square & Degree of freedom & Mean of square \\
\hline Between time & 120086.5 & 2 & 60043.27 \\
\hline Between time and analyst & 120088.5 & 3 & 40029.5 \\
\hline Residual & -120083 & 6 & -20013.8 \\
\hline Total & 120092.3 & 11 & - \\
\hline
\end{tabular}

TABLE 4: Interday precision study for TE.

\begin{tabular}{lccc}
\hline Source of variation & Sum of square & Degree of freedom & Mean of square \\
\hline Between day & 119702.2 & 2 & 59851.08 \\
Between day and analyst & 119701.7 & 3 & 39900.58 \\
Residual & -119694 & 6 & -19949.1 \\
\hline Total & 119709.4 & 11 & - \\
\hline
\end{tabular}


TABLE 5: Robustness study for TE and EM.

\begin{tabular}{lccc}
\hline Factor & Level & \multicolumn{2}{c}{ Mean \% assay, \% RSD } \\
& & TE & $99.34,0.87$ \\
Shaking time $( \pm 2 \mathrm{~min})$ & 10 & $99.2,0.97$ & $98.18,0.25$ \\
& 6 & $99.45,0.43$ & 9.23 \\
Measurement wavelength $( \pm 1 \mathrm{~nm})$ & $260(\mathrm{EM}), 288.9(\mathrm{TE})$ & $99.08,0.48$ & $99.43,0.54$ \\
& $262(\mathrm{EM}), 290.9(\mathrm{TE})$ & $99.09,0.72$ & $100.45,1.34$ \\
Concentration of TE in blank $( \pm 1 \mu \mathrm{g} / \mathrm{mL})$ & 21 & $100.3,0.59$ & $99.78,0.45$ \\
\hline
\end{tabular}

TABLE 6: Results of specificity study for TE $(n=3)$.

\begin{tabular}{lcccc}
\hline Concentration $(\mu \mathrm{g} / \mathrm{mL})$ & Abs. standard & Abs. sample & Difference $(D)$ & 0.00526 \\
\hline 6 & 0.11504 & 0.1203 & -0.0244 & $2.16 \times 10^{-4}$ \\
12 & 0.2478 & 0.2234 & 0.0084 & $2.24 \times 10^{-4}$ \\
18 & 0.3681 & 0.3765 & -0.0097 & $3.19 \times 10^{-4}$ \\
24 & 0.4884 & 0.4787 & -0.0268 & $6.35 \times 10^{-8}$ \\
30 & 0.6087 & 0.5819 & $3.01 \times 10^{-4}$ \\
\hline
\end{tabular}

wavelength of measurement $( \pm 1 \mathrm{~nm})$, and concentration of TE in reference cell $( \pm 1 \mu \mathrm{g} / \mathrm{mL})$.

3.7. Specificity Study. Specificity of the method was determined by comparing the absorbance values of standard mixture of drugs and formulation sample at specified wavelengths for both drugs. Mean of three absorbance values of standard mixture and formulation sample in the concentration range $4-20 \mu \mathrm{g} / \mathrm{mL}$ for $\mathrm{TE}$ and $6-30 \mu \mathrm{g} / \mathrm{mL}$ for EM were compared by $t$-test. Calculated $t$ values $\left(t_{\text {cal }}\right)$ were determined by MIP Pharmasoft 1.0.

\section{Results and Discussion}

4.1. Baseline Manipulation Method. The Beer Lambert law [6] is defined as, when a beam of monochromatic radiation is passed through a solution of absorbing molecules, the rate of decrease of intensity of incident radiation with thickness [1] of the absorbing solution which is proportional to the intensity of incident $\left(I_{0}\right)$ radiation as well as the concentration $(c)$ of the solution and mathematical expression of the law is, $\log I_{0} / I=\varepsilon * c * 1=A$ [1] where $I=$ intensity of transmitted light, $\varepsilon=$ molar absorptivity, $c=$ concentration of solution in moles/litre, $l=$ path length, and $A$ $=$ absorbance $\left(\log I_{0} / I\right)$. In double beam, spectrophotometer blank is used to eliminate the contribution to absorbance by solvents. Under the situation, modified equation applicable is $A_{\text {observed }}=[\varepsilon * c * 1]_{\text {sample }}-[\varepsilon * c * 1]_{\text {blank }}[2]$, and on this basis UV spectrum is obtained. By keeping solution of analytes(s) of appropriate concentration in blank, it is possible to obtain independent wavelength(s) in spectra for each analyte(s) form the mixture which is the basis of the baseline manipulation method. For the simultaneous determination using the baseline manipulation method, mixed standard solutions of TE and EM in linearity range were prepared from standard stock solutions in distilled water. These solutions were scanned in the range of $200-400 \mathrm{~nm}$ by keeping individual standard solution as blank. When TE $20 \mu \mathrm{g} / \mathrm{mL}$ was used as blank linear, response of both analytes was observed. Wavelengths 261 and 289.9 were selected for TE and EM, respectively, instrument responses was measured at the selected wavelengths and used for preparation of calibration curve. It is easier to apply baseline manipulation method when overlay spectra of analytes show well-resolved peaks.

Overlain spectra of TE $(4-20 \mu \mathrm{g} / \mathrm{mL})$ and EM $(6-30 \mu \mathrm{g} / \mathrm{mL})$ in distilled water are presented in (Figure $1)$.

Typical baseline manipulation spectrograph of TE and EM in combination when TE $20 \mu \mathrm{g} / \mathrm{mL}$ was used as blank is shown in (Figure 2).

\subsection{Types of Baseline Manipulation Methods}

4.2.1. Singular Baseline Manipulation (SBM). In this method, composition of blank remains constant throughout the experiment. The method has certain advantages such as less time is required compared to other UV methods, less number of dilutions/solutions is required, and the method is suitable for binary mixtures of analytes.

4.2.2. Multiple Baseline Manipulation (MBM). Composition of the blank is changed to estimate different analytes form the mixture. The method has advantages same as SBM and it can be used for ternary mixtures of analytes. In this method, spectrum of sample containing mixture of three drugs shows two different peaks from which suitable analytical wavelength can be assigned to two analytes which is free from inferences by other analyte in mixture. 
TABLE 7: ANOVA table of comparison of results of reported spectrophotometric and proposed baseline manipulation methods for TE.

\begin{tabular}{lccc}
\hline Source of variation & Sum of square & Degree of freedom & Mean square \\
\hline Between samples & 3.8174 & 3 & 1.272 \\
Within sample & 26.364 & 16 & 1.647 \\
\hline Total & 30.182 & - & - \\
\hline
\end{tabular}

\section{Method Validation}

The newly developed method was validated according to the ICH guidelines with respect to specificity, linearity, accuracy, precision, and robustness.

\subsection{Linearity, Range, and Method Sensitivity (LOD and LOQ).} Both drugs obey Beer's law in the concentration ranges of $4-20 \mu \mathrm{g} / \mathrm{mL}$ for TE and $6-30 \mu \mathrm{g} / \mathrm{mL}$ for EM. Typical regression equations for the calibration curve of analysts are given in Table 1. Calibration curves were plotted as concentration of drugs versus absorbance. In test of linearity, that is, Fisher variance ratio method, calculated value $F\left(F_{\text {cal }}\right)$ was found to be 0.716 for TE and 2.2 for EM. $F_{\text {cal }}$ values for both analytes were less than the tabulated $F$ values $\left(F_{\text {tab }}\right)$, hence the method shows linear characteristics. LOD values were found to be $0.55 \mu \mathrm{g} / \mathrm{mL}$ for TE and $1.5 \mu \mathrm{g} / \mathrm{mL}$ for EM. LOQ values were found to be $1.58 \mu \mathrm{g} / \mathrm{mL}$ for TE and $4.5 \mu \mathrm{g} / \mathrm{mL}$ for EM.

5.2. Formulation Analysis and Accuracy Studies. The assay for the marketed tablets was established with present spectrophotometric condition (band width 0.5 and scan speed $400 \mathrm{~nm}$ per minute) which was developed and it was found to be accurate and reliable. The average drug content was found in the range 99.35 to 101.2 for both drugs in two formulations, that is, formulation I and II. No interfering peaks were found in spectrograph, indicating the estimation of drug free from inference of excipients. Results of recovery study were in the range of $99.5-101.4 \%$ and percentage of RSD was always less than 0.69 . The optical characteristic of the method and results for formulation analysis are presented in Table 1.

5.3. Recovery Studies. The accuracy study was carried as per the procedure described in Section 2. Baseline amount of TE and EM used was 6 and $9 \mu \mathrm{g} / \mathrm{mL}$, respectively. Results of accuracy study are in the range of 99.45 and 101.4 and percentage of RSD values were less than 0.94 . Results of recovery study are presented in Table 2.

5.4. Precision. Precision data evaluated by using experimental design based $F\left(F_{\text {cal }}=0.722\right)$ value of TE was on ANOVA in which calculated were always less than tabulated $F\left(F_{\mathrm{tab}}\right)$ 3.24 values for both the analytes. Results of inter-day and intraday precision study for TE are presented in ANOVA Table 3 and Table 4, respectively. The $F_{\text {cal }}$ values between time and between time and analyst were found to be -3.0001 and -2.0001 , respectively, while $F_{\text {tab }}$ values between time and time and analyst were found to be 5.14 and 4.76 , respectively.
5.5. Robustness. Effect of variation of experimental conditions was studied and results are presents as described previously. Results of the robustness study for TE \& EM are presented in Table 5 . The data shows that the assay and percentage of RSD values were well within the limit.

5.6. Specificity Studies. Specificity was performed as described in the procedure section. The absorbances were measured of the standard mixture of drug and formulation sample at specified wavelength, that is, 261 for TE. Calculated $t$ values $\left(t_{\text {cal }}\right)$ were determined by MIP Pharmasoft 1.0. These values for the standard mixture of drug and formulation sample were less than tabulated $t\left(t_{\mathrm{tab}}\right)$ values. As the $t_{\text {cal }}$ is less than $t_{\mathrm{tab}}$, it not only proves that there is no significant difference between standard mixture of drugs and formulation sample but also proves specificity of method. Overlay spectra of standard mixture and formulation solution is also similar which further proves the specificity of method. Results of specificity study for TE are presented in Table 6. The $t_{\text {cal }} 1.2980$ values of EM are less than that of the $t_{\text {tab }} 2.78$ values.

5.7. Comparison Study. Baseline manipulation method was compared with the laboratory developed first order derivative, ratio derivative method, and absorption corrected method. The results of ANOVA for TE are shown in Table 7. As the $F_{\text {cal }}$ values are less than the $F_{\text {tab }}$ values for both drugs, it can be concluded that there is no significance difference among these methods, and hence the baseline manipulation method is equivalent to these reported methods.

\section{Conclusions}

The developed method was found to be simple, sensitive, accurate, and precise and can be used for the routine quality control analysis of EM and TE. The concept of baseline manipulation method can be extended to other UV active drug molecules in formulation and in combination. As the method could effectively separate responses in spectra of the drugs from each other in a single spectrometric scan, it reduces human efforts and minimizes errors. The baseline manipulation method is equivalent to other methods such as area under curve or simultaneous estimation of drugs or other multicomponent method used for binary combination.

\section{Acknowledgments}

The authors would like to thank Emcure Pharmaceutical Ltd., Pune, for providing gift samples of drugs. Authors are 
also thankful to the Management and Principal of MAEER's Maharashtra Institute of Pharmacy, Pune, for providing necessary facilities. The authors declare that they have no conflict of interests.

\section{References}

[1] N. A. Gomes, V. V. Vaidya, A. Pudage, S. S. Joshi, and S. A. Parekh, "Liquid chromatography-tandem mass spectrometry (LC-MS/MS) method for simultaneous determination of tenofovir and emtricitabine in human plasma and its application to a bioequivalence study," Journal of Pharmaceutical and Biomedical Analysis, vol. 48, no. 3, pp. 918-926, 2008.

[2] H. P. Rang, M. M. Dale, J. M. Ritter, and R. J. Flower, Rang and Dale's Pharmacology, Churchill Livingstone, Elsever, 6th edition.

[3] V. Jullien, J. M. Tréluyer, G. Pons, and E. Rey, "Determination of tenofovir in human plasma by high-performance liquid chromatography with spectrofluorimetric detection," Journal of Chromatography B, vol. 785, no. 2, pp. 377-381, 2003.

[4] V. Bezy, P. Morin, P. Couerbe, G. Leleu, and L. Agrofoglio, "Simultaneous analysis of several antiretroviral nucleosides in rat-plasma by high-performance liquid chromatography with UV using acetic acid/hydroxylamine buffer: test of this new volatile medium-pH for HPLC-ESI-MS/MS," Journal of Chromatography B, vol. 821, no. 2, pp. 132-143, 2005.

[5] T. Delahunty, L. Bushman, and C. V. Fletcher, "Sensitive assay for determining plasma tenofovir concentrations by LC/MS/MS," Journal of Chromatography B, vol. 830, no. 1, pp. 6-12, 2006.

[6] M. E. Barkil, M. C. Gagnieu, and J. Guitton, "Relevance of a combined UV and single mass spectrometry detection for the determination of tenofovir in human plasma by HPLC in therapeutic drug monitoring," Journal of Chromatography B, vol. 854, no. 1-2, pp. 192-197, 2007. 

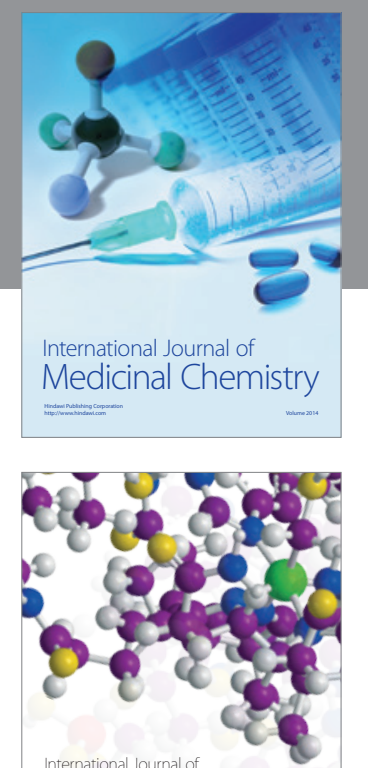

\section{Carbohydrate} Chemistry

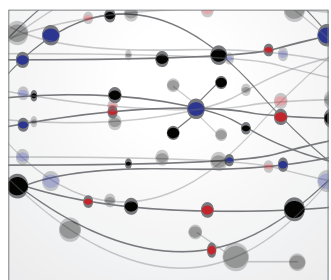

The Scientific World Journal
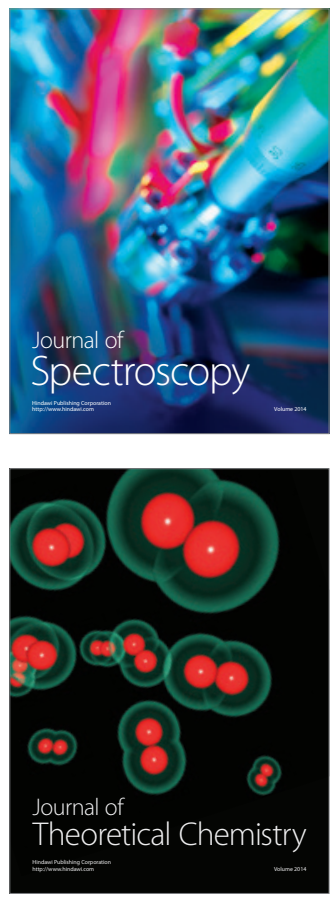
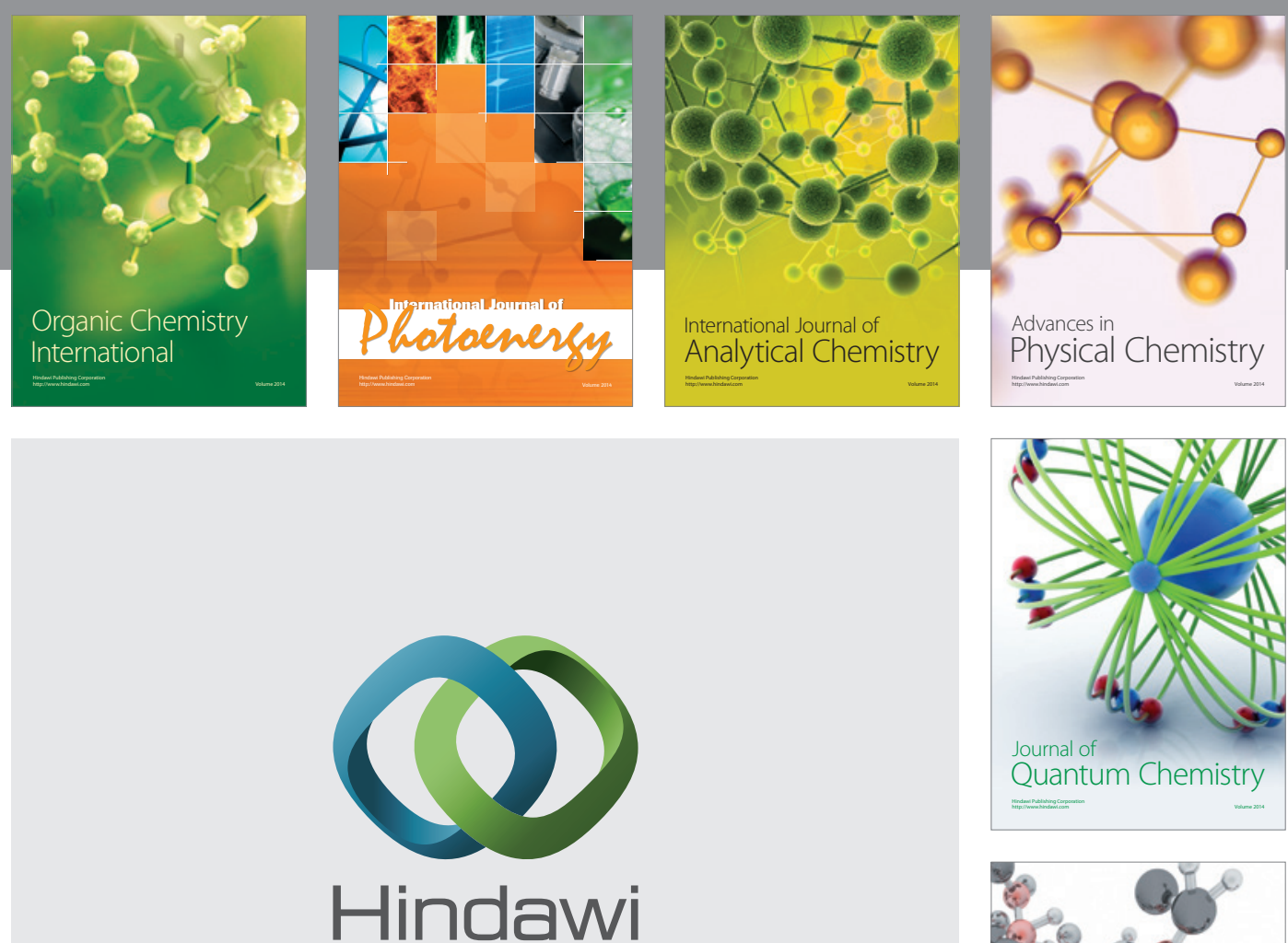

Submit your manuscripts at

http://www.hindawi.com

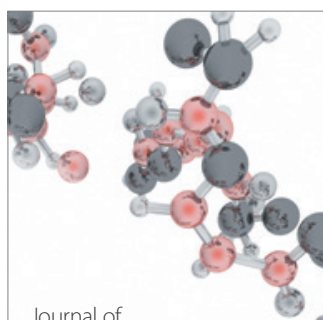

Analytical Methods

in Chemistry

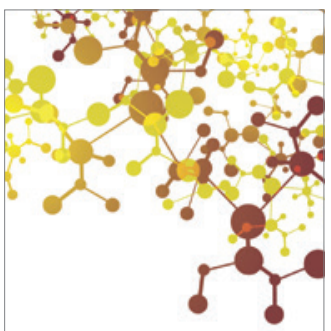

Journal of

Applied Chemistry

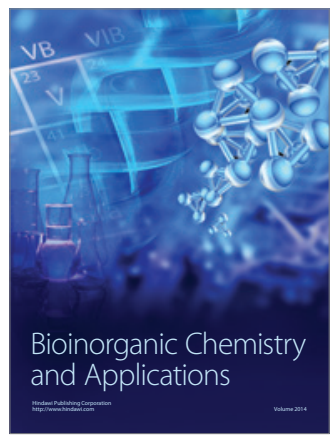

Inorganic Chemistry
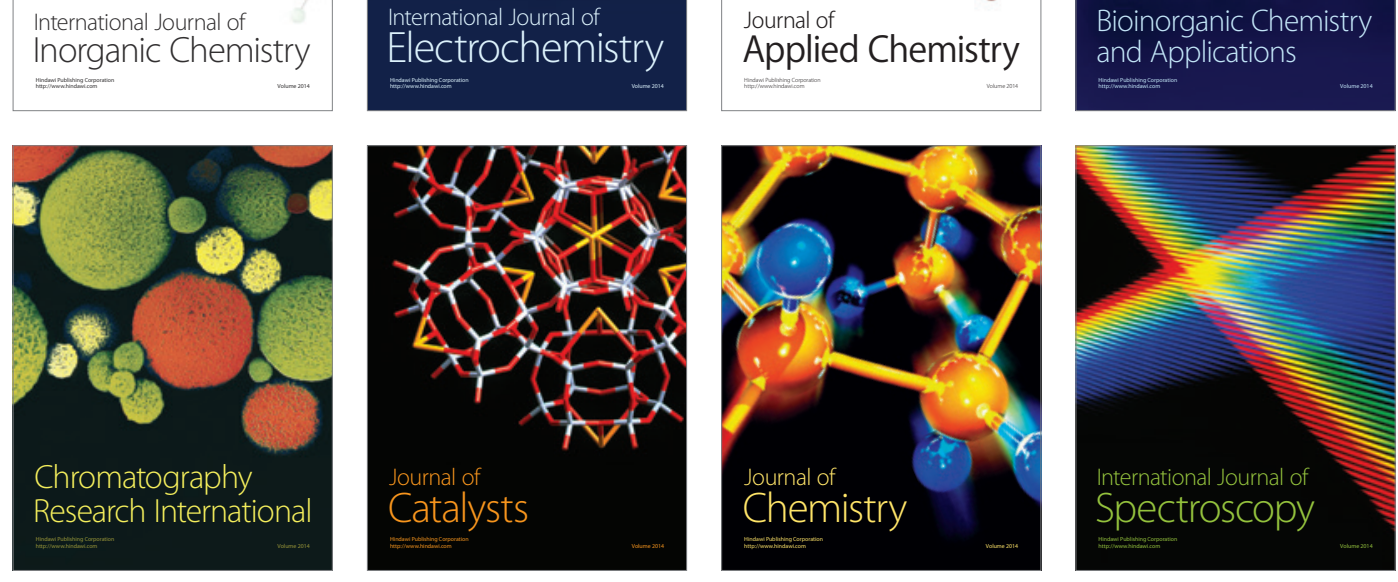